\title{
Factors affecting the slope of empirical ROC curves: Comparison of binary and rating responses'
}

JOSEPH MARKOWITZ AKD JOHN A. SWETS

BOLT BERANEK AND NEWMAN INC., CAMBRIDGE, MASSACHUSETTS

Receiver operating characteristics (ROC curves) were obtained at six signal-to-noise ratios, with two response procedures, in each of three detection paradigms. In all three paradigms-one-interval and two-interval simple detection, and two-interval pedestal-a binary response produced ROC curves with slopes near unity at all signal levels, and a category-rating response led to ROC curves whose slopes decreased with increasing signal strength. This result is thought to be attributable to the varying a priori probability of signal presentation associated with our binary response procedure. The index of detectability taken from the negative diagonal of the ROC space was found to be invariant despite the differences in slope.

The concept of an ROC (Receiver Operating Characteristic) curve, or iso-sensitivity curve, needs little explanation here. In the application of statistical decision theory (Wald, 1950) to signal detection (Peterson, Birdsall, \& Fox, 1954), and in the application of detection theory to human observers (Tanner \& Swets, 1954), it was assumed that a continuous representation of information was avallable on which an observer could base his reports of perceptual events. Moreover, impetus was given to the consideration of errors of commission, or "false alarms," as well as to errors of omission. For these reasons, examination of ROC curves became germane, and deficiencies in the then-current notions of sensory thresholds were noted.

It was convenient to assume at first that the hypothesized distributions of noise and of signal plus noise that underlie the ROC curves were Gaussian and of equal variance. However, it soon became clear that assuming Gaussian distributions of unequal variance (Swets, Tanner, \& Birdsall, 1955, 1961), Rayleigh distributions (Marill, 1956; Jeffress, 1964), or exponential distributions (Egan, Greenberg, \& Schulman, 1961; Green \& Swets, 1966) often yielded better correspondence with the data. Consequently, the focus of interest in ROC analysis has shifted from a single parameter, abstracted as an index of sensitivity (e.g., d'), to concern over the shape of the ROC curve.

Interest in the shape of the ROC curve was also spurred by the resurrection of the threshold concept. Better correspondence to the data was achieved by assuming that threshold crossings could be provoked not only by a signal but by extraneous events as well, and that a response bias leads to reporting some nondetections as being signals or some detections as being non-signals (Luce, 1963a, b). In addition there has been consideration of the possibility of not one fixed threshold, but a number of them, leading to a discrete, multiple-state model for detection (Norman, 1964).

Investigators desirous of drawing inferences about underlying distributions and their continuous or discrete nature are faced with the problem of obtaining better defined ROC curves.

There are a number of ways of obtaining ROC curves, each with their attendant advantages and disadvantages. Consider the experiment with a single observation interval per trial, containing either signal plus noise (s) or noise alone ( $n$ ), where the observer is required to indicate "yes, a signal was present" (S), or "no, a signal was not present" (N). The signal is presented on random trials with a priori probability $P$, and all parameters of the noise background and the signal are held constant. If the observer applies himself to the task consistently, then all theories are agreed that the data from a group of such trials define a single point, $\langle p(S \mid n), p(S \mid n)\rangle$, on an ROC curve. Restricting the observer to the same binary response, additional points on the curve are estimated from different groups of trials in which the observer has been induced to adopt different criteria governing the two responses. This criterion shift can be accomplished by explicit instructions, by changing the a priori probability of signal presentation, or by changing the values and costs assigned to the possible decision outcomes-the so-called "payoff matrix."

It is readily apparent that the adequate definition of a single ROC curve in this manner may be quite time-consuming. A number of distinct points are needed, necessitating a number of groups of trials, each with its associated criterion. Moreover, each group of trials must be necessarily large if the variance of the point is to be satisfactorily small. This is particularly true if the a priori probability of signal presentation is manipulated to produce the various criteria because a large number of trials is then needed to achieve a sufficiently large sample of the less frequent event. For reasons of economy, then, investigators have become increasingly disposed to abandon a simple binary-response procedure and to use in its stead a category-rating procedure (see, e.g., Egan, Schulman, \& Greenberg, 1959).

In the category-rating procedure, the observer has not merely binary response alternatives, but rather 
a multiplicity of response alternatives (e.g., $1,2,3, \ldots$, $m)$. The observer is required to maintain simultaneously a number (m-1) of distinct criteria and to indicate, by the appropriate response alternative, between which two criteria an observation lies. The category-rating procedure may be interpreted as finding discrete approximations to the two continuous density functions hypothesized to underlie the detection process. The cumulative distribution functionswhich provide the coordinates for the ROC plot-are similarly piece-wise estimated by simple summation from the density approximations.

The economy of this procedure is, of course, theory bound, depending upon the assumption that the observations and the criteria are orderable along some dimension-say, likelihood ratio. A notable exception is the two-state theory of Luce $(1963 a, b)$ which, while proposing a metric for the criteria, specifically denies that one exists on the observation.

Granting the economy of a rating procedure and cognizant of its basis in theory, the question is whether the binary and rating procedures are empirically equivalent-that is, do they produce identical ROC curves for identical signal parameters? Heretofore the weight of evidence has been that they do. Egan, Schulman, and Greenberg (1959) compared the performance of three subjects in the binary and rating procedures for a single set of signal parameters, and obtained similar ROC curves in the two procedures. The binary ROC curves were obtained by changing the instruction set rather than by introducing an explicit payoff matrix or by varying the a priori probability of signal presentation, and the rating procedure utilized four categories. The detection paradigm they used may be called "one-interval simple-detection"-a single observation interval is defined in each trial, and the observer is to detect the presence or absence of a pulsed sinusoid against a background of Gaussian noise.

The present experiment provides further comparisons of ROC curves obtained by rating and binary responses. In this case the binary ROC curves were swept out by varying the a priori probability of signal presentation. Six different signal levels were used in order to ascertain the relation between the slope of the ROC curve (on normal-normal coordinates) and the magnitude of the signal. This is the function of interest if the hypothesized distributions which underlie the detection process have variance unequal, and dependent on their mean separation-as is true of the hypothesized unequal-variance-Gaussian, Rayleigh, and exponential distributions. Whereas Gaussian distributions of equal variance result in ROC curves of unit slope for all signal magnitudes, the other three distributions lead to slopes that decrease from unity as the signal strength increases.

In addition to the one-interval simple-detection paradigm, a procedure providing two observation intervals, with the signal present in one only, was used.
We shall refer to this as the "two-interval" procedure. (We avoid the designation "two-altemative forcedchoice." When a rating response is solicited, the choice is among more than two alternatives. Of course, whenever a binary response is solicited, the observer is forced to choose from among two alternatives, even in the one-interval paradigm.) The idea of using a category-rating response in the two-interval procedure has recently been exploited by Schulman and Mitchell (1966). At two different signal levels they compared rating ROC curves obtained in both the one-interval and two-interval paradigms.

In the present experiment six signal levels were used in the two-interval paradigm, with binary as well as rating responses. With both kinds of response two different kinds of background were used. One of the backgrounds was Gaussian noise alone (simple detection). The other background consisted of Gaussian noise and a "pedestal." The pedestal was a clearlyaudible sinusoid, with the same frequency and phase as the signal, pulsed during the observation interval; the signal in this case was an increment in the pedestal.

\section{PROCEDURE}

The signal was a $1000-\mathrm{Hz}$ sinusoid of $100-\mathrm{msec}$. duration, gated at a positive-going zero-crossing. The continuous background of Gaussian noise had a spectrum level of approximately $50 \mathrm{~dB}$ re $0.0002 \mathrm{~d} / \mathrm{cm}^{2}$. The pedestal was presented at $E / N_{O}=32.2$, where $E$ is the signal energy, and $N_{o}$ is the noise-power density. The stimuli were presented through earphones.

A digital computer (Digital Equipment Corporation PDP-8) was used for command and control purposes. It was responsible for all timing, information to the observers, pseudo-random implementation of the a priori probabilities, data acquisition, and rudimentary data reduction. All audio stimuli were of analogue origin, but their presentation was controlled by the computer via multiplex switches.

When a binary response was required, three a priori probabilities were used. These probabilities-of signal presentation in the one-interval paradigm, or of the signal appearing in the first interval in the two-interval paradigm-were $0.20,0.50$, and 0.80 . In all conditions in which a rating response was solicited, the a priori probability was .50 .

Four rating categories were used. In the one-interval procedure they were defined as follows: (1) confident the signal was present, (2) think the signal was present but with lower confidence, (3) think the signal was absent, but not confident, and (4) confident the signal was absent. It was explained to the observers that this could be interpreted as two sequential binary decisionsone as to presence or absence, the other as to confidence in the first decision-or, alternatively, as simply a four-point rating scale of certainty about signal presence. The four response alternatives were similarly defined in the two-interval procedure, with "interval 1" and "interval 2" substituted for "present" 


\begin{tabular}{|c|c|c|c|c|}
\hline Condition & $\begin{array}{l}\text { Detection } \\
\text { Paradigm }\end{array}$ & $\begin{array}{c}\text { Response } \\
\text { Type }\end{array}$ & $\begin{array}{l}\text { A Priorl } \\
\text { Probab1l1ty }\end{array}$ & $\begin{array}{l}\text { No. of Trials } \\
\text { per signal Level }\end{array}$ \\
\hline 1 & $\begin{array}{l}\text { One-Interval } \\
\text { Simpie Detection }\end{array}$ & Rating & .5 & 1000 \\
\hline 2 & $\begin{array}{l}\text { One-Interval } \\
\text { Simple Detection }\end{array}$ & Binary & $.2, .5, .8$ & 2400 \\
\hline 3 & $\begin{array}{l}\text { Two-Interval } \\
\text { Simple Detection }\end{array}$ & Rating & .5 & 1000 \\
\hline 4 & $\begin{array}{l}\text { Two-Interval } \\
\text { S1mple Detection }\end{array}$ & Binary & $.2, .5, .8$ & 2400 \\
\hline 5 & $\begin{array}{l}\text { Two-Interval } \\
\text { Pedestal }\end{array}$ & Rating & .5 & 1000 \\
\hline 6 & $\begin{array}{l}\text { Two- Interval } \\
\text { Pedestal }\end{array}$ & Binary & $.2, \quad .5, .8$ & 2400 \\
\hline
\end{tabular}

Table 1. Summary of the Six Experimental Conditions (Six Signal Levels Were Used in Each Condition.) and "absent." The observers were told that the use of the categories should be relative to the signal level so that categories would be used approximately equally often for each level.

Warning, observation, and answer intervals were defined for the observers by indicator lights. Following each trial, the observers were informed as to whether, or in which interval, a signal had been presented on that trial. The observers were female high-school seniors. Daily sessions of two hours contained six biocks of trials, 200 trials per block, with brief rest periods between blocks.

Three observers served simultaneously through six experimental conditions, over a period of about 14 weeks. The first two conditions used the one-interval simple-detection procedure; a rating response was made in Condition 1 and a binary response was made in Condition 2. The next two conditions used the twointerval simple-detection procedure, with a rating response in Condition 3 and a binary response in Condition 4. The last two conditions used the twointerval-pedestal procedure, with a rating response in Condition 5 and a binary response in Condition 6.

Six signal levels were presented in each condition. In the rating-response conditions $(1,3,5)$, each signal level was presented for a block of 200 trials in each session, with order randomly determined. Five sessions per condition thus provided an ROC curve defined by 1000 trials at each signal level. In the binaryresponse conditions $(2,4,6)$ each of the three a priori probabilities was in effect for four consecutive 200-trial blocks. Data were collected at all three probabilities for a given signal level before the level was changed; with that restriction the signal levels were ordered randomly, as were the three probabilities at each signal level. Twelve sessions per condition thus produced an ROC curve defined by 2400 trials at each signal level.

The signal levels in the simple-detection conditions were $\mathrm{E} / \mathrm{N}_{\mathrm{O}}=4.4,6.8,9.7,13.1,17.0$, and 21.4 , and, in the pedestal conditions, $\Delta \mathrm{E} / \mathrm{N}_{\mathrm{O}}=2.4,13.0,21.9$, $31.6,38.5$, and 45.7 .

A summary of the experimental conditions is given in Table 1. We have only to add that Condition 1 was preceded by four days of practice, and each of the other conditions by one day of practice, and that one observer withdrew from the experiment after Condition 5.

\section{RESULTS AND DISCUSSION}

ROC curves were plotted on normal-normal coordinates for each subject, each condition, and each signal level, and are included in the Appendix. Two parameters were then abstracted from each curve, the slope and the sensitivity index $d_{e}{ }^{\prime}$-where $d_{e}{ }^{\prime}$ is defined as twice the normal deviate of the intersection of the ROC curve and the negative diagonal. These parameters are tabulated by subject, condition, and signal level in Table 2.

If we inspect the slope of the ROC curve as a function of signal strength, a difference between the binary and rating conditions emerges irrespective of the particular detection paradigm-one-interval-simple, two-interval-simple, or two-interval-pedestal. For the three rating conditions, slope is a decreasing function of signal strength. For the three binary conditions, the slope remains relatively constant and near 1.0. This is easily seen in Fig. 1, which shows the average slope as a function of ordinal signal strength. The triangles represent the rating data (Conditions 1,3 , and 5 ), and the circles represent the binary data (Conditions 2,4 , and 6 ).

Figures $2(a, b, c)$ show these same data broken down according to the detection paradigm used-oneinterval-simple, two-interval-simple, or two-intervalpedestal. Note that the non-monotonicity of the rating data observed in Fig. 1 shows up in each paradigm. Observation of Table 2 shows that the non-monotonicity is characteristic of each subject.

The observed difference between binary and rating ROC curves is all the more striking because of the interpretation of the slope as the ratio of the standard deviations of the two distributions hypothesized to underlie the detection process. Consider first the binary ROC curves in the one-interval simple-detection paradigm (Condition 2). These curves, straight lines of close to unit slope, are consonant with the 


\begin{tabular}{|c|c|c|c|c|c|c|c|c|c|}
\hline \multicolumn{2}{|c|}{ Cond1tion } & \multicolumn{2}{|c|}{ Observer 1} & \multicolumn{2}{|c|}{ Observer 2} & \multicolumn{2}{|c|}{ Observer 3} & \multicolumn{2}{|c|}{ Average } \\
\hline \multirow[t]{2}{*}{1} & $\mathrm{E} / \mathrm{N}_{0}$ & $\mathrm{~d}_{\mathrm{e}}^{\prime}$ & slope & $a_{e}^{\prime}$ & slope & $\mathrm{d}_{\mathbf{e}}$ & slope & $\mathrm{d}_{\mathrm{e}}^{\prime}$ & Blope \\
\hline & $\begin{array}{r}4.4 \\
6.8 \\
9.7 \\
13.1 \\
17.0 \\
21.4 \\
\end{array}$ & $\begin{array}{r}.38 \\
.68 \\
1.12 \\
1.72 \\
2.04 \\
2.56 \\
\end{array}$ & $\begin{array}{l}.98 \\
.92 \\
.73 \\
.82 \\
.77 \\
.58 \\
\end{array}$ & $\begin{array}{r}.08 \\
.44 \\
.60 \\
1.04 \\
1.42 \\
1.88 \\
\end{array}$ & $\begin{array}{l}1.02 \\
.78 \\
.73 \\
.86 \\
.78 \\
.70\end{array}$ & $\begin{array}{r}.22 \\
.22 \\
.60 \\
1.02 \\
1.48 \\
1.92 \\
\end{array}$ & $\begin{array}{l}.87 \\
.87 \\
.78 \\
.81 \\
.80 \\
.75 \\
\end{array}$ & $\begin{array}{r}.23 \\
.45 \\
.77 \\
1.26 \\
1.65 \\
2.12 \\
\end{array}$ & $\begin{array}{l}.96 \\
.86 \\
.75 \\
.83 \\
.78 \\
.68 \\
\end{array}$ \\
\hline \multirow[t]{2}{*}{2} & $\mathrm{E} / \mathrm{N}_{0}$ & & & & & & & & \\
\hline & $\begin{array}{r}4.4 \\
6.8 \\
9.7 \\
13.1 \\
17.0 \\
21.4 \\
\end{array}$ & $\begin{array}{r}.44 \\
.86 \\
1.00 \\
1.64 \\
2.00 \\
2.60 \\
\end{array}$ & $\begin{array}{r}.90 \\
.72 \\
1.10 \\
.81 \\
.95 \\
.81 \\
\end{array}$ & $\begin{array}{r}.06 \\
.46 \\
.54 \\
.96 \\
1.06 \\
1.46 \\
\end{array}$ & $\begin{array}{r}.92 \\
.83 \\
.90 \\
.91 \\
.91 \\
1.00 \\
\end{array}$ & $\begin{array}{r}.26 \\
.66 \\
.76 \\
1.46 \\
1.70 \\
2.36 \\
\end{array}$ & $\begin{array}{l}.78 \\
.69 \\
.84 \\
.75 \\
.81 \\
.70 \\
\end{array}$ & $\begin{array}{r}.25 \\
.66 \\
.77 \\
1.35 \\
1.59 \\
2.14 \\
\end{array}$ & $\begin{array}{l}.89 \\
.75 \\
.95 \\
.82 \\
.89 \\
.84\end{array}$ \\
\hline \multirow[t]{2}{*}{3} & $\mathrm{E} / \mathrm{N}_{0}$ & & & & & & & & \\
\hline & $\begin{array}{r}4.4 \\
6.8 \\
9.7 \\
13.1 \\
17.0 \\
21.4\end{array}$ & $\begin{array}{r}.60 \\
.92 \\
1.36 \\
2.60 \\
2.66 \\
3.60\end{array}$ & $\begin{array}{r}.90 \\
.91 \\
.93 \\
.98 \\
1.21 \\
.39\end{array}$ & $\begin{array}{r}.28 \\
.28 \\
.52 \\
.68 \\
1.22 \\
1.42 \\
\end{array}$ & $\begin{array}{l}.91 \\
.56 \\
.76 \\
.71 \\
.54 \\
.44\end{array}$ & $\begin{array}{r}.06 \\
.32 \\
.94 \\
1.52 \\
1.98 \\
2.80\end{array}$ & $\begin{array}{r}1.06 \\
.93 \\
.83 \\
.78 \\
.42 \\
.65\end{array}$ & $\begin{array}{r}.31 \\
.51 \\
.94 \\
1.60 \\
1.95 \\
2.61\end{array}$ & $\begin{array}{l}.96 \\
.80 \\
.84 \\
.82 \\
.72 \\
.49\end{array}$ \\
\hline \multirow[t]{2}{*}{4} & $E / N_{0}$ & & & & & & & & \\
\hline & $\begin{array}{r}4.4 \\
6.8 \\
9.7 \\
13.1 \\
17.0 \\
21.4\end{array}$ & $\begin{array}{r}.56 \\
.88 \\
1.36 \\
-.5 \\
2.52 \\
2.90\end{array}$ & $\begin{array}{c}1.09 \\
.94 \\
.86 \\
-- \\
1.07 \\
1.24\end{array}$ & $\begin{array}{r}.18 \\
.40 \\
.62 \\
.94 \\
1.26 \\
1.58\end{array}$ & $\begin{array}{r}.92 \\
.88 \\
.90 \\
.71 \\
1.23 \\
1.08\end{array}$ & $\begin{array}{l}.28 \\
.62 \\
1.10 \\
1.76 \\
2.26 \\
3.12\end{array}$ & $\begin{array}{r}.99 \\
.84 \\
.92 \\
1.13 \\
.89 \\
1.09\end{array}$ & $\begin{array}{r}.34 \\
.63 \\
1.03 \\
1.35 \\
2.01 \\
2.53\end{array}$ & $\begin{array}{r}1.00 \\
.89 \\
.89 \\
.92 \\
1.06 \\
1.14\end{array}$ \\
\hline \multirow[t]{2}{*}{5} & $\Delta \mathrm{E} / \mathrm{N}_{0}$ & & & & & & & & \\
\hline & $\begin{array}{r}2.4 \\
13.0 \\
21.9 \\
31.6 \\
38.5 \\
45.7\end{array}$ & $\begin{array}{r}0.00 \\
.78 \\
1.54 \\
2.40 \\
2.46 \\
2.56\end{array}$ & $\begin{array}{r}.97 \\
1.01 \\
.82 \\
.86 \\
.84 \\
.56\end{array}$ & $\begin{array}{r}.12 \\
.60 \\
.78 \\
.90 \\
1.02 \\
.86\end{array}$ & $\begin{array}{r}1.13 \\
1.03 \\
.52 \\
.69 \\
.64 \\
.48\end{array}$ & $\begin{array}{r}.16 \\
.80 \\
1.18 \\
1.58 \\
2.26 \\
2.06\end{array}$ & $\begin{array}{r}1.09 \\
.69 \\
.65 \\
.62 \\
.48 \\
.56\end{array}$ & $\begin{array}{r}.09 \\
.73 \\
1.17 \\
1.63 \\
1.91 \\
1.83\end{array}$ & $\begin{array}{r}1.06 \\
.91 \\
.66 \\
.72 \\
.65 \\
.53\end{array}$ \\
\hline 6 & $\begin{array}{r}\Delta E / N_{0} \\
2.4 \\
13.0 \\
21.9 \\
31.6 \\
38.5 \\
45.7\end{array}$ & $\begin{array}{l}.22 \\
1.06 \\
1.76 \\
2.36 \\
2.76 \\
3.10\end{array}$ & $\begin{array}{r}1.04 \\
1.42 \\
1.20 \\
1.11 \\
1.95 \\
1.00\end{array}$ & & & $\begin{array}{r}.04 \\
.90 \\
1.48 \\
2.12 \\
2.40 \\
2.56\end{array}$ & $\begin{array}{r}.97 \\
.86 \\
1.08 \\
1.47 \\
.93 \\
.95\end{array}$ & $\begin{array}{r}.13 \\
.98 \\
1.62 \\
2.24 \\
2.58 \\
2.83\end{array}$ & $\begin{array}{r}1.01 \\
1.14 \\
1.14 \\
1.29 \\
.94 \\
.98\end{array}$ \\
\hline
\end{tabular}

Table 2.

Obtained Values of $d_{e}{ }^{\prime}$ and Slope assumption that the underlying distributions, signal and noise, are Gaussian and of equal variance. However, these curves of unit slope contrast with the ROC curves obtained in the same one-interval simpledetection paradigm, but using a rating response. The rating curves (Condition 1), of generally decreasing slope as a function of signal strength, belie the assumption that the underlying distributions of signal and noise are of equal variance.

Now consider the two-interval simple-detection experiment. The usual theoretical treatment (see, e.g., Green \& Swets, 1966; Luce, 1963; and Schulman \& Mitchell, 1966) of the two-interval paradigm is to assume that the observer's strategy is to take an observation in each interval and compare them. For example, the observer might base his decision upon the difference between the two observations. Under these conditions, we expect two-interval ROC curves to be of unit slope-even if the one-interval curves are not. Moreover, if (and only if) the underlying distributions of signal and noise are of equal variance-as estimated, say, in the one-interval experiment-we expect an index of detectability such as $d_{e}^{\prime}$ to be greater in the two-interval experiment than in the oneinterval experiment by a factor of $\sqrt{2}$ given the same signal-to-noise ratio.

In fact, the binary-response two-interval ROC curves (Condition 4) are of unit slope in accordance with the

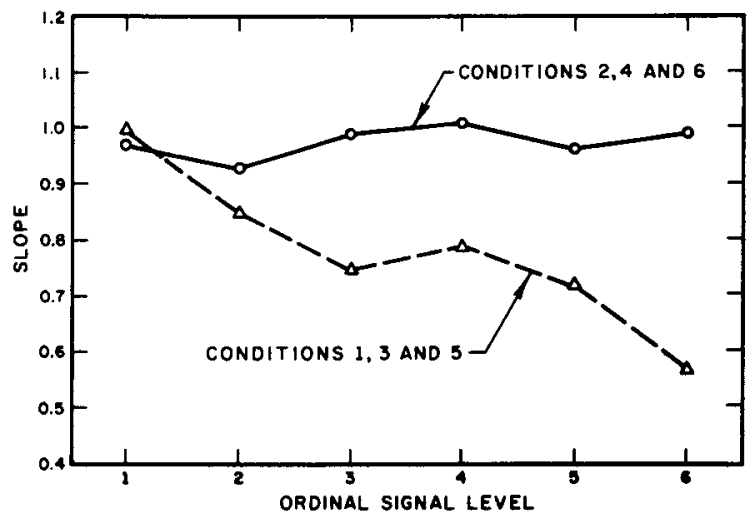

Fig. 1. Slope as a function of ondinal afmal level. Data from binary responses (even conditions) are shown by circles. Data from rating responses (odd conditions) are shown by triangles. 

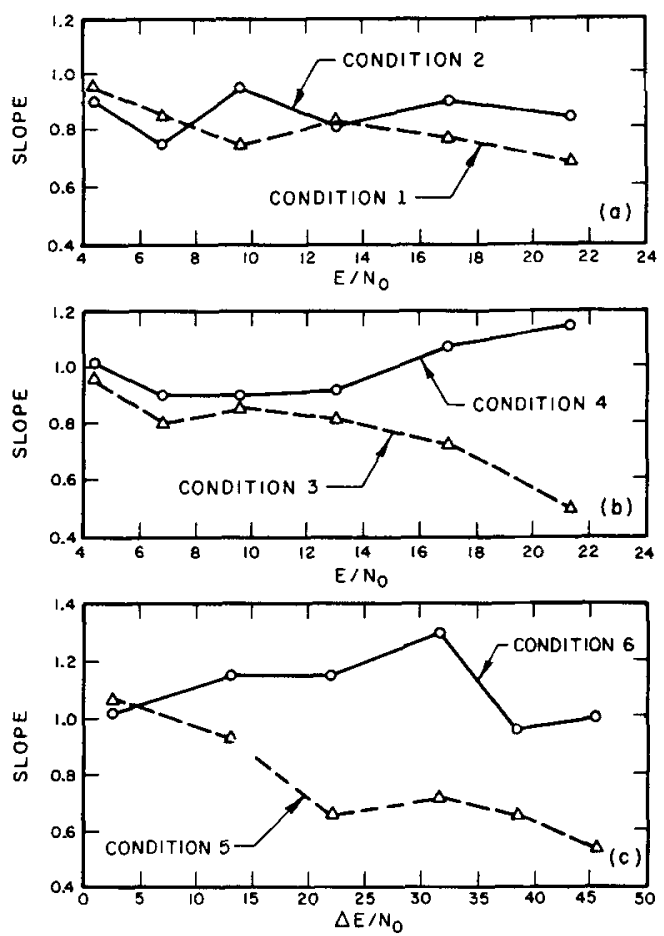

Fig. 2. Slope as a function of signal strength broken down by detection paradigm: (a) one-interval simple-detection, (b) twointerval simple-detection, (c) two-interval pedestal. Data from binary responses (even conditions) are shown by circles. Data from rating responses (odd conditions) are shown by triangles.

standard theoretical interpretation. However, the detectability in the two-interval paradigm exceeds that in the comparable one-interval case (Condition 2) by a factor of less than 1.20 , on the average. The implication of this factor being substantially less than $\sqrt{2}$ is that, if the observer's strategy accords with the assumption, then the underlying distributions of signal and noise cannot be of equal variance. Thus, there is an internal inconsistency in the binary ROC curves. There are two ways to resolve this conflict. One way is to assume that the observer's strategy is not as usually assumed, and we shall have occasion to return to this point. The second resolution is to treat the binary ROC curves in the one-interval paradigm as suspect. And, of course, the disagreement between the binary and rating ROC curves in the one-interval paradigm reinforces this view.

However, if we look at the rating ROC curves for the two-interval simple-detection paradigm (Condition 3) we find slope to be a decreasing function of signal strength. Now this finding, as pointed out above, is at odds with the standard interpretation. These curves should be of unit slope, even though the underlying distributions of signal and noise are of unequal variance-as inferred from Condition 1-provided of course that the observer's strategy is as assumed. Moreover, this finding is in apparent contradiction to the results of Schulman and Mitchell (1966) who found rating ROC curves of approximately unit slope at two signal strengths. Thus, while the results of Schulman and Mitchell (1966) indicate that observers can act in accord with the usual assumption, our observers apparently did not.

The possibility that our observers used strategies not posited by standard theory is reinforced by the slopes of the ROC curves obtained in the two-intervalpedestal condition. The addition of a pedestal should have the effect of driving the distributions of signal and noise closer to equal-variance Gaussian distributions (Green \& Swets, 1966). Nonetheless, the rating ROC curves in this situation (Condition 5) are not of unit slope. The binary ROC curves for the pedestal condition (Condition 6) are, again, of unit slope.

The observed differences between the binary and rating procedures serve to confound those questions concerning the types of distributions hypothesized to underlie the detection process. However, with regard to estimating a parameter of detectability-an end toward which ROC curves are often directed-the prospects are better.
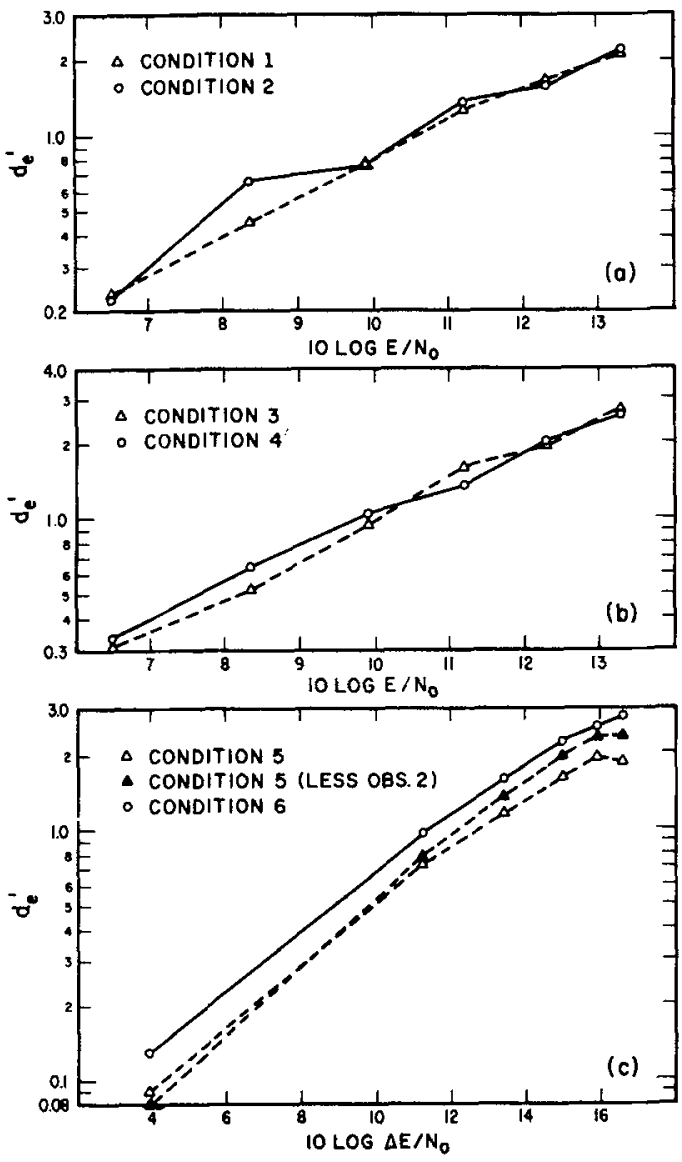

Fig. 3. Psychometric functions for each detection paradigm: (a) one-interval simple-detection, (b) two-interval simple -detection, (c) two-interval pedestal. Data from binary responses (even conditions) are shown by circles. Data from rating responses (odd conditions) are shown by triangles. In Condition 5, closed triangles omit observer 2 and are most comparable to Condition 6 in which observer 2 did not participate. 
As indicated, we have chosen to abstract $d_{e}$ ' as our index of detectability. We might have chosen $\Delta \mathrm{m}$, the $x$ (normal deviate) intercept of the ROC curve, as considered by Green and Swets (1966). Theoretically, $\Delta \mathrm{m}$ might have been the better choice since it is interpreted as the mean separation of the underlying distributions, normalized by the standard deviation of one (i.e., the noise) distribution and unconfounded by the variance of the other (i.e., the signal) distribution. The measure $\mathrm{d}_{e}$, on the other hand, is normalized by the average of the two variances. However, for practical reasons $d_{e}$ 'is superior.

If we plot psychometric functions $-\mathrm{d}_{\mathrm{e}}$ 'vs. $10 \mathrm{log}$ $\mathrm{E} / \mathrm{N}_{\mathrm{O}}$ - for each detection paradigm, as shown in Figs. $3(a, b, c)$, we see that the values of $d_{e}$ from ratingand binary-response procedures are virtually identical, all differences being less than $1 \mathrm{~dB}$. Figure 3a compares average rating (triangles) and binary (circles) psychometric functions in the one-interval simple-detection experiment. Figure $3 b$ shows a similar comparison for the two-interval simple-detection experiment. Likewise, Fig. 3c shows the comparison for the two-interval-pedestal experiment, except that two rating curves are shown, the closed triangles omitting observer 2 who did not participate in the binary-response condition. Of course, given slope differences between the binary and rating curves, a correspondence in $\mathrm{d}_{\mathrm{e}}{ }^{\prime}$ precludes a correspondence of $\Delta \mathrm{m}$.

Thus, the advantage of $\mathrm{d}_{\mathrm{e}}$ ' over $\Delta \mathrm{m}$ is the correspondence of $d_{e}{ }^{\prime}$ for the two types of response, binary and rating. Schulman and Mitchell (1966) have proposed using the ortho-normal distance from the origin to the curve as a measure of detectability claimed to be unconfounded by the variances of the underlying distributions-hence independent of the slope. Simple trigonometric considerations show very little discrepancy between the measures for fair departures from slope $=1$. For slopes of, say, 0.7, the difference is $1.5 \%$. Thus the Schulman-Mitchell index of detectability would, like $\mathrm{d}_{\mathrm{e}}{ }^{\prime}$, be preferred to $\Delta \mathrm{m}$. Note, however, that $\mathrm{d}_{\mathrm{e}}{ }^{\prime}$ can be obtained from a single point on the ROC curve, provided it lies on the negative diagonal, by referring to tables such as those of Elliott (1959) or Blosser (1965).

In summary; we have found differences between the ROC curves produced with rating responses and those produced with binary responses. These differences are most marked in a two-interval paradigm. As a measure of detectability $d_{e}^{\prime}$ seems satisfactory inasmuch as it seems to be independent of the type of response used.

\section{CONCLUSION}

The data present two general problems. Suppose for the moment that we accept the data of Condition 1the rating data in a one-interval simple-detection setting-as accurately describing the underlying distributions of signal and noise. Then we see that as the mean separation increases the ratio of the variance of the signal distribution to that of the signal noise distribution increases. This phenomenon is generally referred to as a mean-to-sigma ratio. However, the previously-noted non-monotonicity precludes fitting our data with a constant mean-to-sigma ratio. The lower several signal strengths appear to have a mean-to-sigma ratio of about three, and the higher signals are better fitted with a mean-to-sigma ratio of six. Also because of the lack of a monotonic relation, distributions like the Rayleigh and the exponential would fail to provide satisfactory fits.

A second problem we must face is why the twointerval rating data are not of slope one. To resolve this problem we would have to assume two distributions $f(s)$ and $f(n)$ and a binary operation, $\oplus$, such that $f(s \odot n) \neq f(n \odot s)$.

But now let us see why the binary-response procedure might not accurately portray the underlying distributions if the rating procedure does. Recall that the binary ROC curves were obtained by varying the a priori probability of signal presentation. Thus the expected time since last a signal was presented is inversely proportional to the presentation probability. If there is a degradation in performance as this time is increased, then the three points which we have been supposing to lie on a single ROC curve would lie on separate curves, as shown in exaggerated fashion in Fig. 4 . In fact, Schulman and Greenberg (1960) have shown that points obtained with different a priori probabilities may indeed lie on different curves. This hypothesis is open to future experimental test in several ways. First, binary ROC curves could be obtained by varying costs and values while keeping a priori probabilities fixed. Second, rating ROC curves could be obtained at various a priori

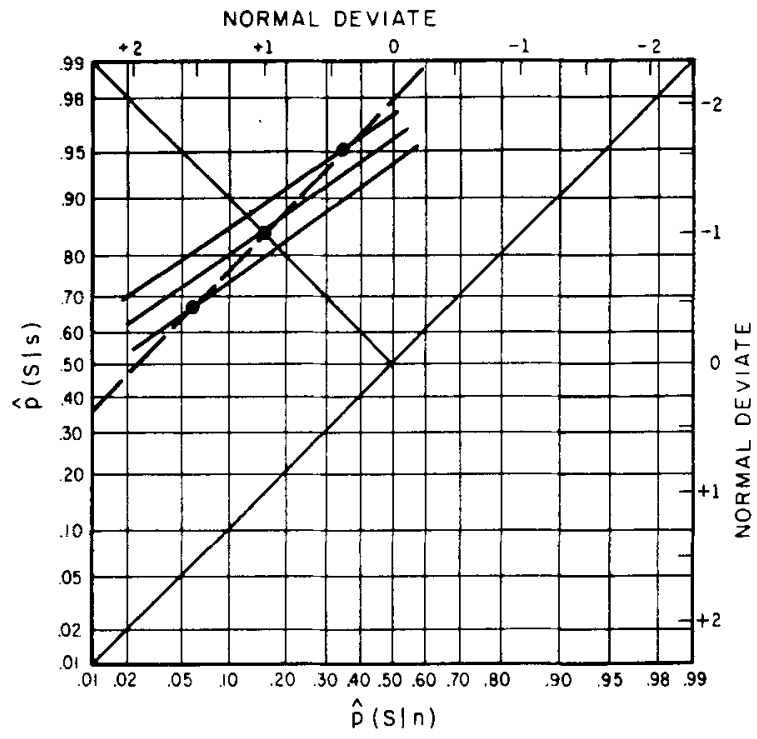

Fig. 4. An exaggerated depiction of how three distinct ROC curves of slope $<l$ (solid lines) might be combined into a single curve of steeper slope (dashed line). The solid curves are assumed to be associated with different a priori probabilities of signal presentation, but identical signal-to-noise ratios. 


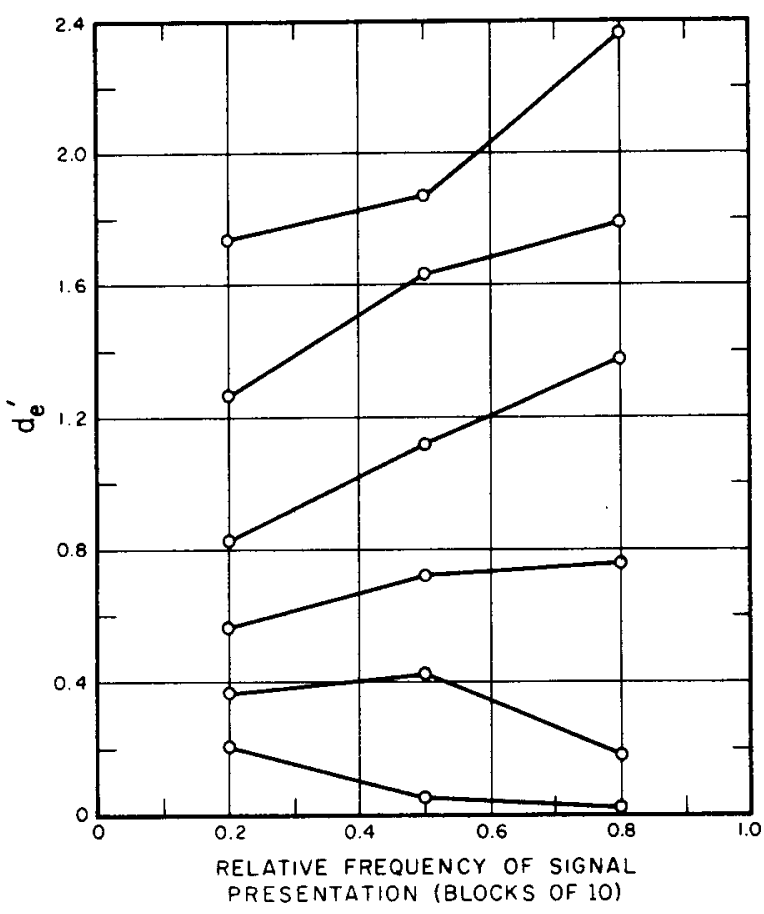

Fig. 5. Average values of $d_{e}$ as a function of relative frequency of signal occurrence for six signal levels (data of Condition 1).

probabilities. Finally, a signal cue might precede each trial to remove the inverse variation of a priori probabilities and expected time since last signal.

We can get some insight about the assumption of separate ROC curves for different a priori probabilities by subjecting certain of the present data to further analysis. Although the rating data in the one-interval simple-detection paradigm (Condition 1) were collected at an a priori probability of 0.50 , it was possible to select 10 -trial blocks in which the ratio of signal to non-signal trials was $20: 80$ and $80: 20$ as well as 50:50. In this way we were able to plot three ROC curves (of analyzed a priori probabilities 0.20 , 0.50 , and 0.80 ) for each observer, for each signal level. The index of detectability, $d_{e}$ ', was abstracted from the curves, and Fig. 5 shows the average $d_{e}$ as a function of analyzed a priori probability, for the six signal levels.

Reference to Fig. 4 suggests that we would expect higher $\mathrm{d}_{\mathrm{e}}{ }^{\prime}$ values for higher a priori probabilities. As shown in Fig. 5, $d_{e}{ }^{\prime}$ does increase with increasing frequency of signal presentation, at least at the higher signal-to-noise ratios. The average data are repre- sentative of each subject, and the size of the effect. is large as sequential effects go. Of course, further experimental tests remain to be done, as indicated above, but it does seem as if various a priori probabilities of signal presentation will yield distinct ROC curves.

\section{References}

Blosser, A. B. A performance-oriented approach to detection: Tables for detection, discrimination, and decision theory. Tracor Inc. Document No. Tracor 65-267-U, August, 1965.

Egan, J. P., Greenberg, G. Z., \& Schulman, A. I. Operating characteristics, signal detectability, and the method of free response. J. Acoust. Soc. Amer., 1961, 33, 993-1007.

Egan, J. P., Schulman, A. 1., \& Greenberg, G. Z. Operating characteristics determined by binary decisions and by ratings. $J$. Acoust. Soc. Amer., 1959, 31, 768-773.

Elliott, P. B. Tables of d'. Tech. Report, 97, University of Michigan: Electronic Defense Group, Ann Arbor, 1959.

Green, D. M., \& Swets, J. A. Signal detection theory and psychophysics. New York: Wiley, 1966.

Jeffress, L. A. Stimulus-oriented approach to detection, J. Acoust. Soc. Amer., 1964, 36, 766-774.

Luce, R. D. A threshold theory for simple detection experiments. Psychol. Rev., 1963a, 70, 61-79.

Luce, R. D. Detection and recognition. In R. D. Luce, R. R. Bush and E. Galanter (Eds.), Handbook of mathematical psychology. New York: Wiley, 1963b. Pp. 103-189.

Marill, T. M. Datection theory and psychophysics. Massachusetts Institute of Technology: Research Laboratory of Electronics, Tech. Report No. 319, 1956.

Noman, D. A. Sensory thresholds, response biases, and the neural quantum theory. J. Math. Psychol., 1964, 1, 88-120.

Peterson, W. W., Birdsall, T. G., \& Fox, W. C. The theory of signal detectability. Trans. IRE Professional Group on Information Theory. PGIT-4, 171-212, 1954.

Schulman, A. I., \& Greenberg, G. Z. Operating characteristics and a priori probability of the signal. J. Acoust. Soc. Amer., 1960 , 32,1505 (A).

Schulman, A. I., \& Mitchell, R. R. Operating characteristics from yes-no and forced-choice procedures. J. Acoust. Soc. Amer., $1966,40,473-477$.

Tanner, W. P., Jr., \& Swets, J. A. A decision-making theory of visual detection. Psychol. Rev., 1954, 61, 401-409.

Swets, J. A., Tanner, W. P., Jr., \& Birdsall, T. G. The evidence for a decision-making theory of visual detection. University of Michigan: Electronic Defense Group. Tech. Report No. 40, 1955.

Swets, J. A., Tanner, W. P., Jr., \& Birdsall, T. G. Decision processes in perception. Psychol. Rev., 1961, 68, 301-340.

Wald, A. Statistical decision functions. New York: Wiley, 1950.

\section{Note}

1. This work was supported by the Human Performance Branch of the NASA-Ames Research Center under Contract No. NAS2-2676. Linda S. McElroy and Susan Levin collected the data and performed analyses of them, and their contributions are gratefully acknowledged.

(Accepted for publication December 5, 1966.) 


\section{Appendix}

ROC curves for each observer by condition. Different symbols serve only to associate those points run with a common signal-tonoise ratio, where confusion might otherwise result.

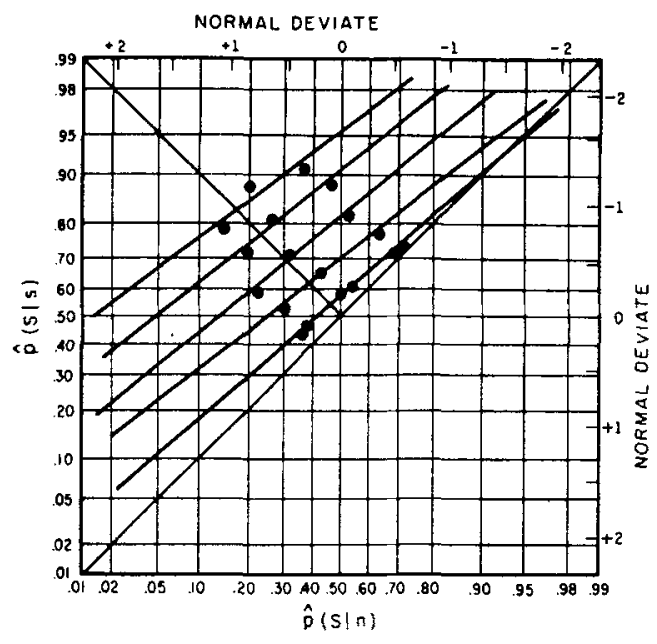

OBSERVER 3 CONDITION 1

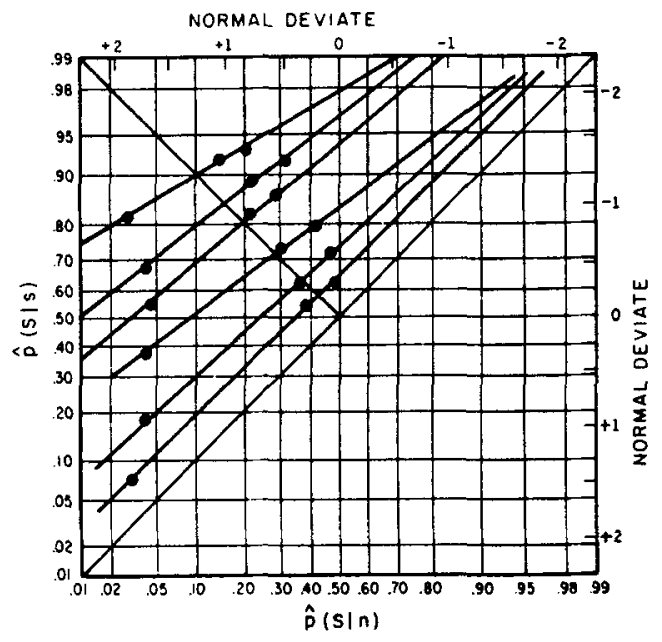

OBSERVER 1

CONOITION I

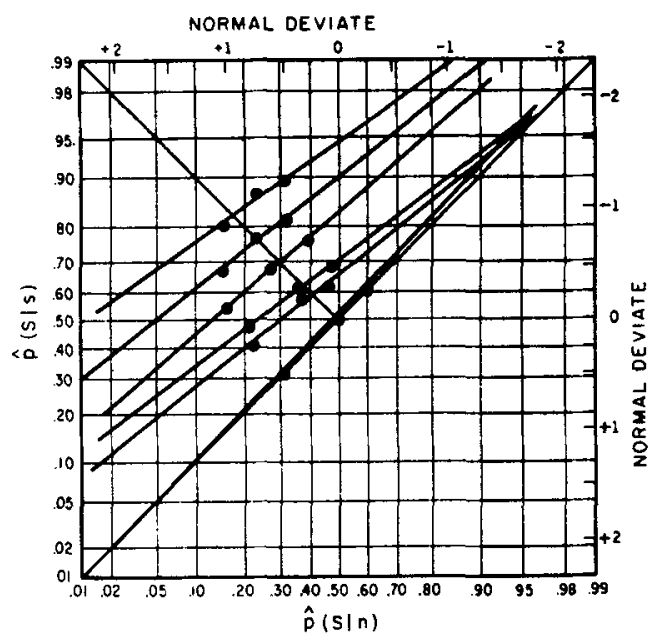

OBSERVER 2

CONDITION 1

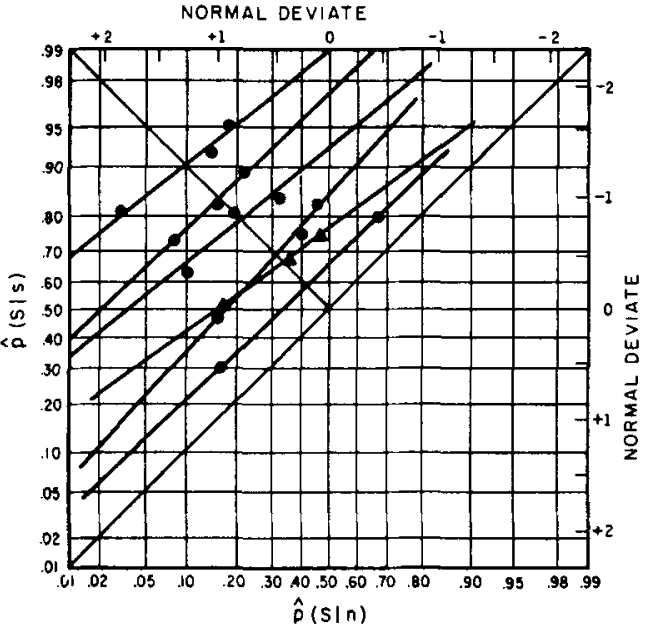

OBSERVER 1

CONDITION 2

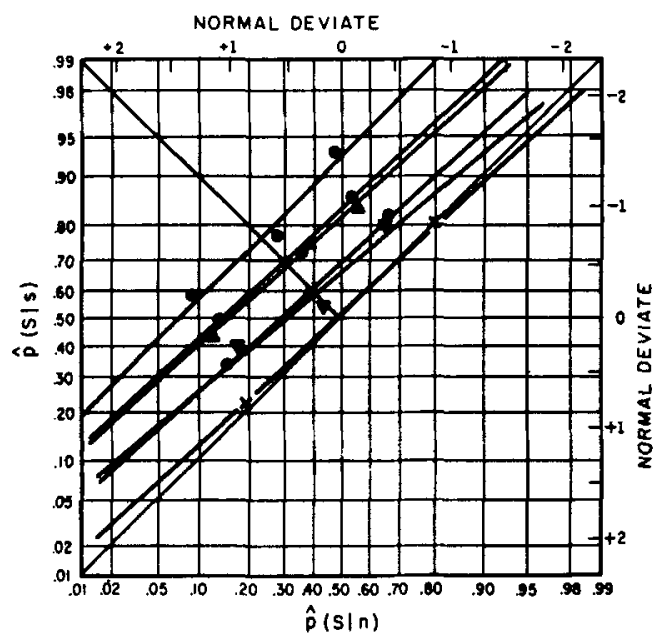

OBSERVER 2

CONDITION 2 


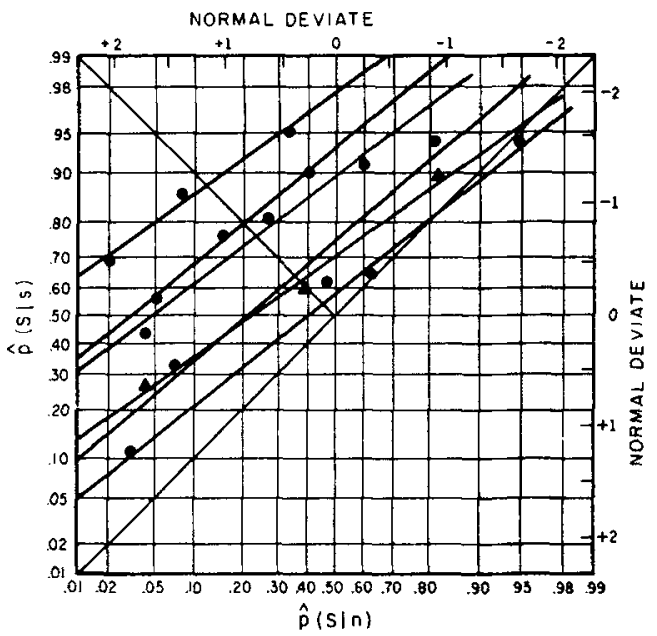

OBSERVER 3

CONOITION 2

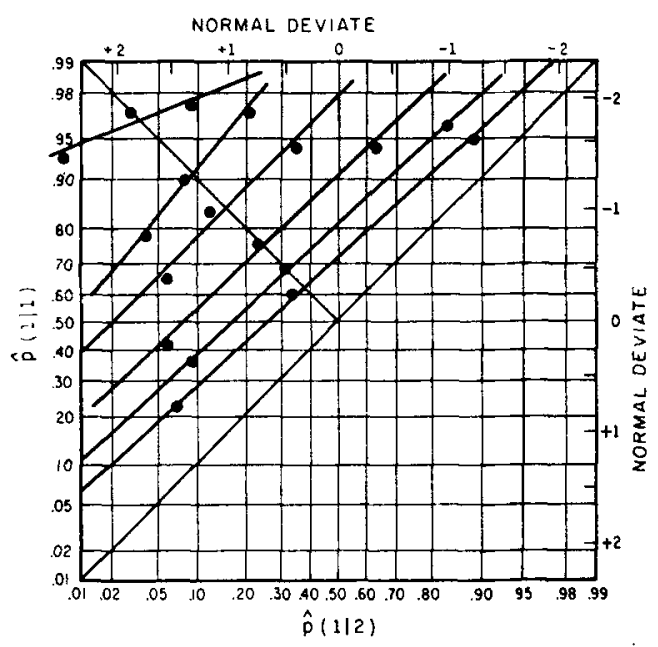

OQSERVER I

CONDITION 3

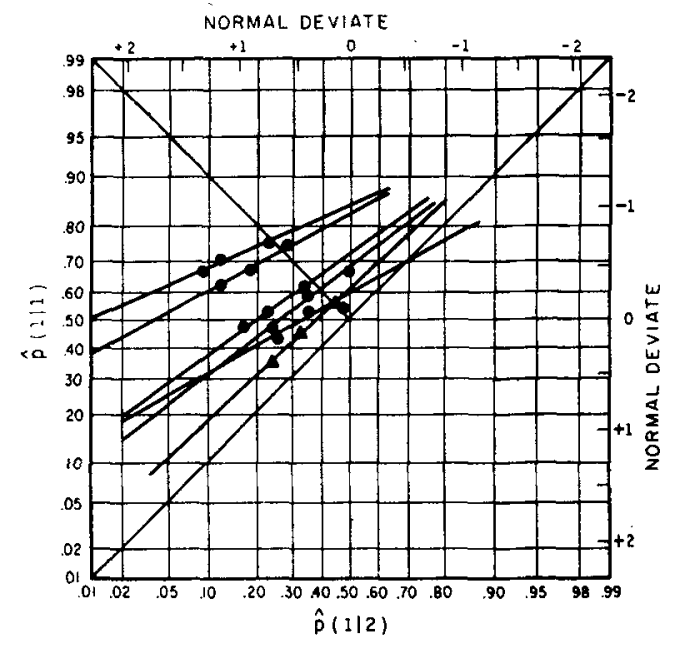

OBSERVER 2

CONDITION 3

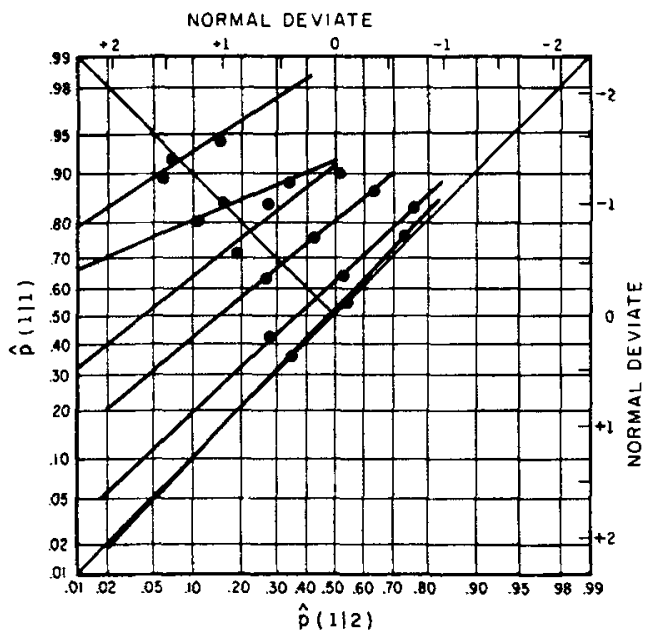

OBSERVER 3

CONDITION 3

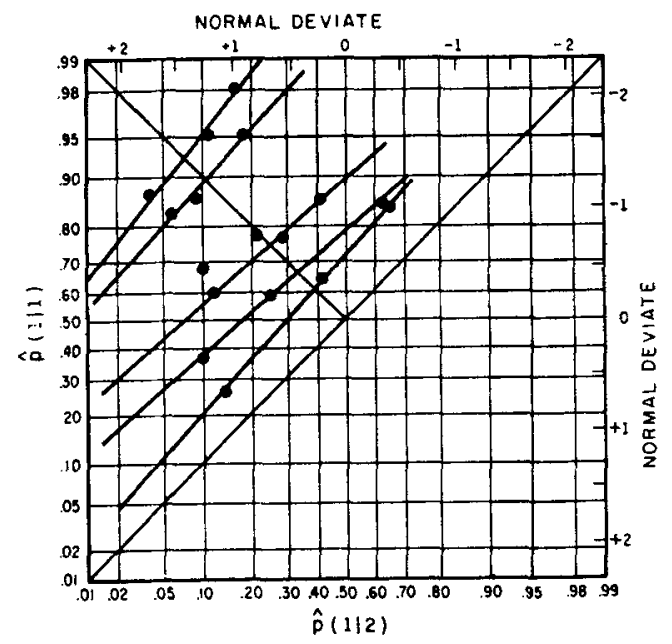

OBSERVER 1

CONOITION 4

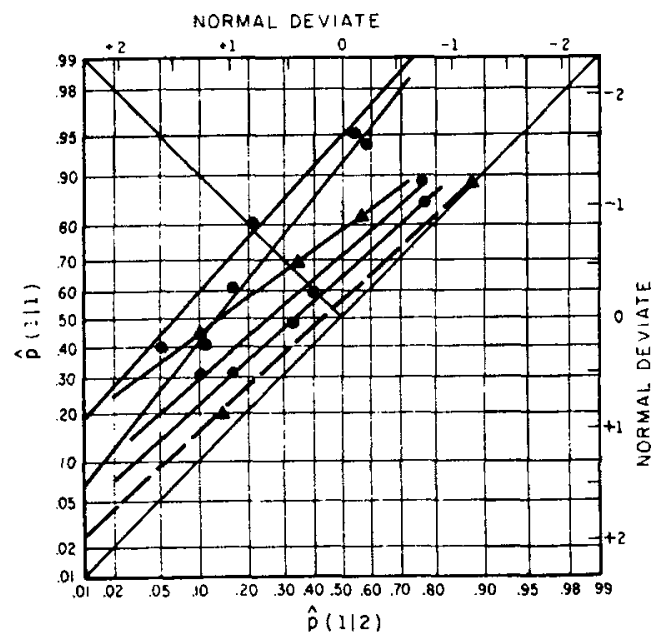

OBSERVER 2

CONDITION 4 


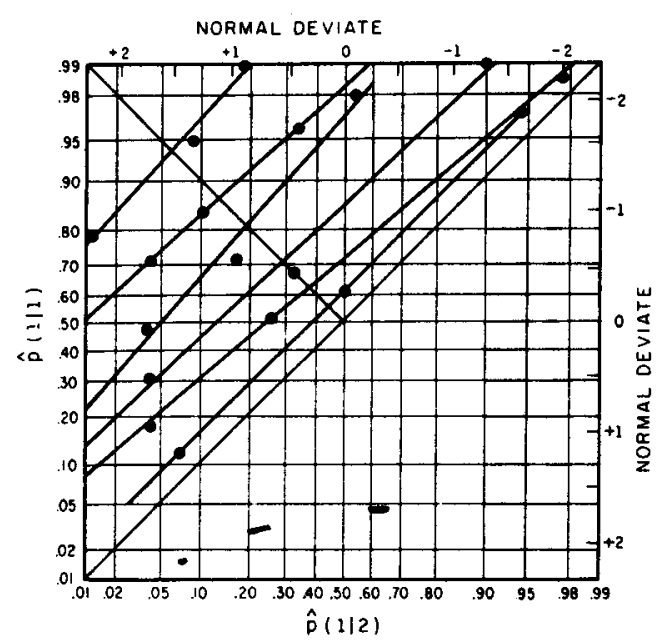

OBSERVER 3 CONDITION 4

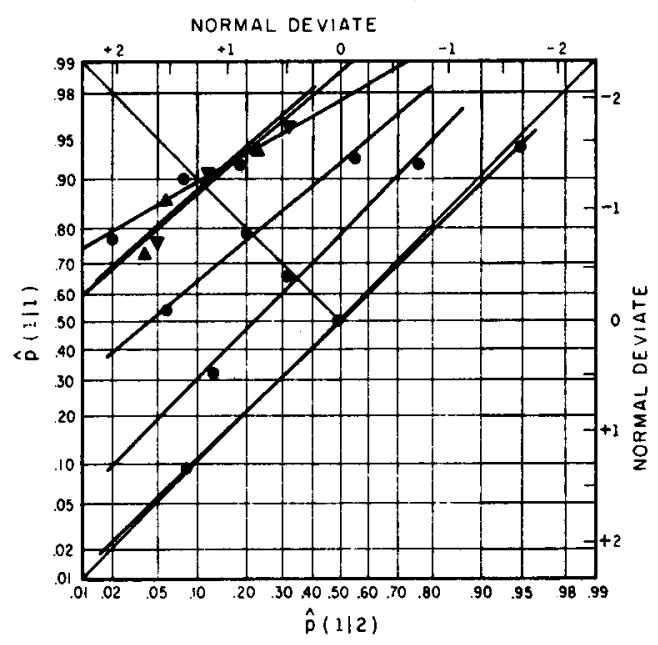

OBSERVER I

CONOITION 5

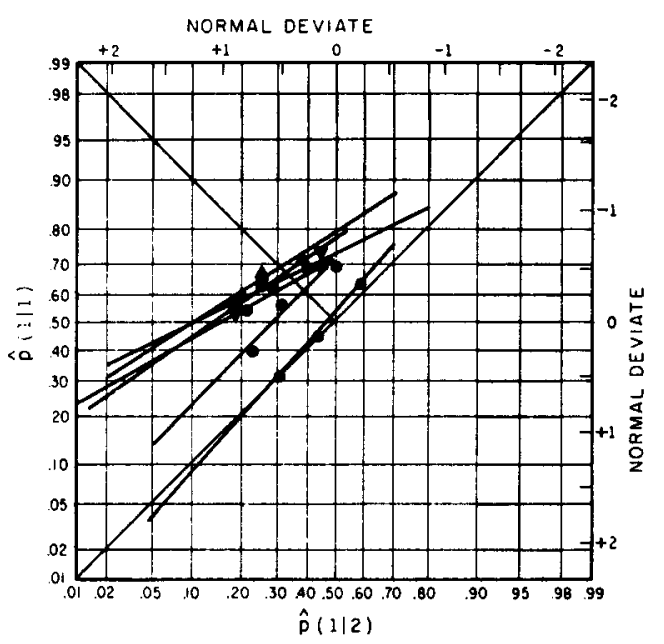

OBSERVER 2

CONUITION 5

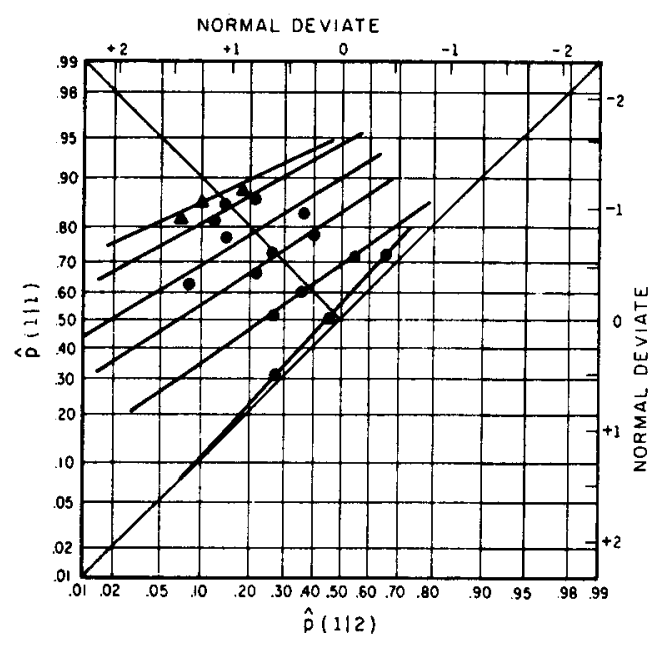

OBSERVER 3

CONDITION 5

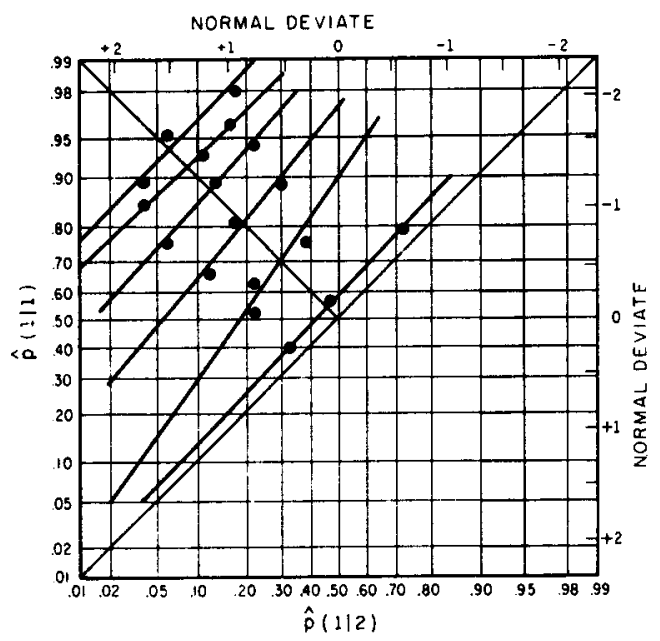

OBSERVER 1

CONDITION 6

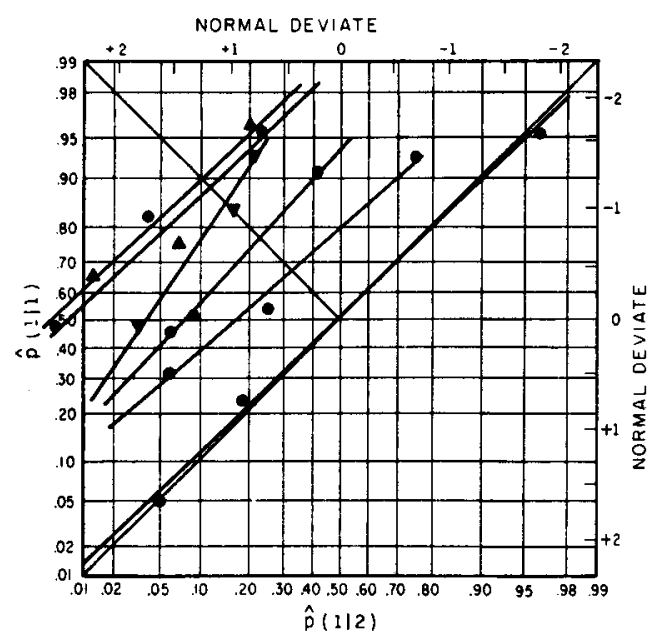

OBSERVER 3

CONDITION 6 